\section{Influence of Mastitis and Repeat Breeding Incidence on Participation in the Animal Insurance Program for Dairy Farmers in Ba Vi, Hanoi, Vietnam}

\author{
Kentaro Koide ${ }^{1}$, Ryo Murata ${ }^{1}$, Au Xuan Khoa ${ }^{2}$, Nguyen Khanh \\ Ly$^{2}$, Phan Thi Tam ${ }^{2}$, Vu Thi Thu Tra ${ }^{3}$, Duong Van Nhiem ${ }^{3}$, \\ Satoko Kubota ${ }^{4}$, Hiroichi Kono ${ }^{4} \&$ Kohei Makita ${ }^{1}$
}

\author{
${ }^{1}$ School of Veterinary Medicine, Rakuno Gakuen University, Bunkyodai Midorimachi, \\ Ebetsu, Hokkaido, 069-8501, Japan \\ ${ }^{2}$ National Center for Veterinary Diagnosis, Hanoi, Vietnam \\ ${ }^{3}$ Faculty of Veterinary Medicine, Vietnam National University of Agriculture, Hanoi \\ 131000, Vietnam \\ ${ }^{4}$ Obihiro University of Agriculture and Veterinary Medicine, Inada-cho, Obihiro, Hokaido, \\ 080-8555, Japan
}

\begin{abstract}
The livestock insurance program for dairy farmers in Vietnam targeting various acute diseases began in 2011 as a pilot project; however, due to vaccination campaigns, outbreaks of such diseases are rare. The objective of this study was to investigate the effects of mastitis and reproductive failure-which chronically affect the farming economy-on the decision to participate in the insurance program. A survey involving a questionnaire and milk sampling from apparently healthy animals was conducted on the 38 participating and 59 non-participating dairy farms in the livestock insurance program in $\mathrm{Ba} \mathrm{Vi}$, Hanoi, Vietnam, in August 2014. Microbiological tests were performed to detect sub-clinical mastitis, whereas the questionnaire was administered to collect information regarding farm management and the occurrence of clinical mastitis and reproductive failure over the previous three months. There were no significant differences in the proportion of farms having cows with clinical mastitis (insured: $8 / 38$ farms, $21.1 \%$ vs. non-insured: 13/58 farms, $\mathrm{NA}=1,22.8 \%, \mathrm{p}=1$ ) and repeat breeders (insured: 16/36 farms, NA $=2,44.4 \%$ vs. non-insured: $20 / 57$ farms, $\mathrm{NA}=2,35.1 \%, \mathrm{p}=0.49$ ) over the previous three months. The proportion of farms having cows with sub-clinical mastitis at the time of the survey was also not significantly different between insured (5/38 farms, $13.2 \%)$ and noninsured $(7 / 59$ farms, $11.91 \%)$ farms $(p=1)$. Based on the obtained results, neither endemic disease nor farm management is respectably involved in the decision of farms to participate in the animal insurance program targeting acute animal infectious diseases.
\end{abstract}

\section{Keywords}

Animal insurance, decision, bovine mastitis, repeat breeding, Vietnam 


\section{Introduction}

Milk production has drastically increased over the last four decades, particularly in Southeast Asia, including Vietnam (Food \& Agricultural Organization, 2013). This growth has been attributed to an increase in the domestic demand for dairy products and the governmentled importation of highly productive dairy animals for breeding purposes (Garcia et al., 2006). A comparison with the average milk yield in 1997 indicated that due to genetic improvements in the lactating ability of Vietnamese dairy cows, one Vietnamese cow produces as much milk as four dairy cows in India, the second-largest milk-producing country in the world. Proper nutritional management is necessary for high milk-producing cows because of their sensitive energy balance (Grant \& Albright, 1995). The risk factors for diseases in dairy cows are interconnected (LeBlanc et al., 2006), and rapid changes in dairy productivity are assumed to have increased the incidence of various diseases, including sub-clinical forms of mastitis, ketosis, rumen acidosis, and endometritis.

In addition to the increased production and consumption of livestock products in Southeast Asia, rapid economic growth has been accompanied by increased risks of infectious disease epidemics such as foot-and-mouth disease (FMD) (Vu et al., 2017) and avian influenza (Poolkhet et al., 2018), primarily due to increases in the frequency and quantity of trade and travel. As the majority of livestock producers in Southeast Asia are small scale, the economic impact of such outbreaks is immense.

One method of dealing with the risk of disease outbreaks is insurance. In Japan, agricultural insurance for dairy farms is subsidized $50 \%$ by the government and covers not only the death of animals but also treatment costs (Japanese Ministry of Agriculture Forestry \& Fisheries, 2010). Moreover, under the Act on Domestic Animal Infectious Diseases Control, the Japanese government compensates farmers for $80 \%$ of the value of animals culled due to several important infectious diseases, such as FMD, and the remaining $20 \%$ of the value is compensated by the insurance program for participating farmers (Kadowaki et al., 2016). Crop and livestock insurance was first introduced in Vietnam between 1985 and 1987 (phase 1), without government support. However, except for crude rubber insurance, phase 1 was withdrawn in 1987 due to problems associated with moral hazards and conflicts regarding pay-out value (Kono et al., 2017). By order of the Vietnamese prime minister, dairy insurance premiums were implemented in 2011 within the phase 2 pilot program for three years, covering rice plants, livestock (buffaloes, cows, pigs, and chickens), and aquatic products (catfish, tiger prawns, and white-leg shrimp) (Kono et al., 2017). Under the pilot program involving commercial insurance companies, the state-subsidized $100 \%$ of the insurance premiums for poor farming households and individuals, initially $80 \%$ and later $90 \%$ for near-poor, $60 \%$ for the remaining, and $20 \%$ for relevant farming organizations (Duc, 2017). Three causes of death were covered by the dairy insurance: FMD, anthrax, and Pasteurella infection, but no treatment costs were covered for endemic and non-infectious production diseases. Vaccination campaigns against FMD, anthrax, and Pasteurella infection are being implemented in dairy production areas of Vietnam (personal communication with local veterinary officers), and animal deaths due to these diseases appear to be rare. The $\mathrm{Ba} \mathrm{Vi}$ district of Hanoi is an important dairy production area in Vietnam and characterized by advanced dairy technology. As of October 2013, 260 farms in $\mathrm{Ba} \mathrm{Vi}$ were participating in the insurance program (Kono et al., 2017). The phase 2 pilot insurance program was successful, with a high proportion $(76.8 \%)$ of poor households in the target area participating. However, the program also had several weaknesses, including a lack of testing and certification facilities for diseases, resulting in limited compensation or farmers receiving inconsistent damage assessments (Duc, 2017). The government, therefore, considered instituting a public-private partnership (Duc, 2017) covering $90 \%$ of insurance premiums for poor and nearpoor farming households and $20 \%$ for remaining households (Viet Nam News, 2018). 
Mastitis has been described as the most expensive disease affecting dairy farms worldwide; however, the chronic nature of this disease can lead farmers to ignore and/or underestimate the financial effects of decreased milk production and increased culling (Hogeveen et al., 2011). Mastitis is caused by a wide variety of pathogenic bacteria. Microorganisms causing mastitis are classified as either contagious or environmental pathogens, depending on the vector of transmission. The major contagious pathogens are Staphylococcusaureus and Streptococcus agalactiae, S. aureus is reportedly the most problematic udder pathogen in bovine mastitis in many parts of the world (Roberson et al., 1994). In southern Vietnam, mastitis is commonly caused by $S$. agalactiae, a contagious organism associated with sub-clinical mastitis (Keefe, 1997; Östensson et al., 2013). In Ba Vi, a $25 \%$ prevalence of sub-clinical mastitis was reported (Suzuki et al., 2006), but much higher prevalences $(63.2 \%$ at quarter, and $88.6 \%$ at cow levels) were reported in southern Vietnam (Lam, 2011).

Repeat breeders are cows that have failed to conceive after three or more services. Repeat breeders are characterized by a normal estrus cycle length, no abnormalities in vaginal discharge, and no palpable abnormalities in the reproductive tract (Bartlett et al., 1986; Gustafsson \& Emanuelson, 2002). A repeat breeder cow appears to be normal, and it is, therefore, difficult to diagnose the cause of the failure to conceive. Repeat breeding results in a delay in starting lactation and fewer calves per cow; therefore, total farm milk yield is also reduced, causing substantial economic losses to farmers. A study involving Holstein and Lai Sind crossbred cattle in Ho Chi Minh city found that reproductive performance was satisfactory in heifers but poor for cows due to declining body condition scores during the first months of lactation and at insemination (Nguyen-Kien et al., 2017), suggesting the potential for improvement in feed management.

This study aimed to determine whether the occurrence of chronic and expensive diseases such as mastitis and repeat breeding affected the decision-making process regarding participation in the phase 2 dairy insurance scheme among dairy farmers in a dairy-production area of Vietnam.

\section{Materials and Methods}

\section{Study site}

This study was conducted in two communes, Yen Bai and Van Hoa, located in Ba Vi, Hanoi (Figure 1). This area, located in northern Vietnam, has a humid tropical climate, characterized by monsoons. Summers (May to July) are very hot, with an average temperature over $27^{\circ} \mathrm{C}$, whereas winters (November to January) are cold and relatively dry (Garcia et al., 2006). This survey was carried out in August 2014. The average precipitation in August is the highest of the entire year, at over $300 \mathrm{~mm}$. Precipitation in winter is low, but these communes are at or near a lake, and extensive irrigation and pumping systems have been established (Garcia et al., 2006).

\section{Sampling and questionnaire survey}

Sample size was calculated for a comparison of two proportions, with the confidence level set at $95 \%$ and the power set at $80 \%$ (Equation 1), in order to primarily compare the prevalence of subclinical mastitis at the animal level between insured and non-insured farms (Dohoo et al., 2014). In equation $1, Z_{\alpha}$ is a multiplier for type I errors, alpha $=0.05,1.96 ; Z_{\beta}$ is a multiplier for type II errors = $0.2 ; \mathrm{p}$ is the expected prevalence, 0.25 (Suzuki et al., 2006); $\mathrm{q}$ is the expected prevalence complement, $0.75 ; \mathrm{p} 1$ is the expected prevalence at insured farms, 0.35; and $\mathrm{p} 2$ is the expected prevalence at non-insured farms, 0.15 , assuming a $20 \%$ difference in the prevalence of sub-clinical mastitis. The sample size was adjusted for a clustering effect assuming an intra-class correlation coefficient of 0.1 (Dohoo et al., 2014).

$$
\frac{\left(z_{\alpha} \sqrt{2 p q}-z_{\beta} \sqrt{p_{1} q_{1}+p_{2} q_{2}}\right)^{2}}{\left(p_{1}-p_{2}\right)^{2}} \text { (Equation 1) }
$$

The sample size was calculated at 73 ; however, 95 farms were involved in the study. The sample size was proportionally allocated between Yen Bai and Van Hoa communes to reflect the total number of insured dairy farmers 


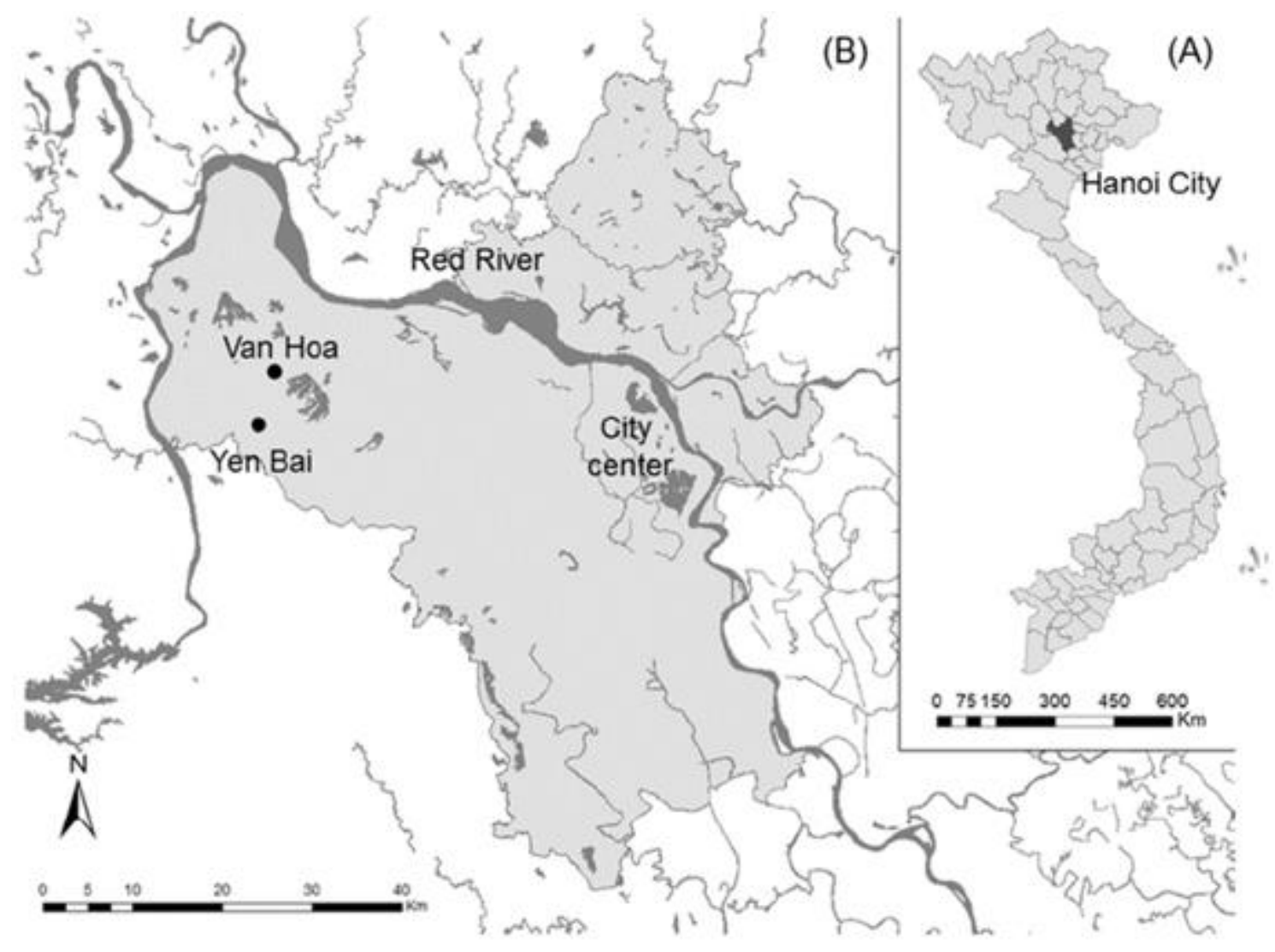

Figure 1. Study areas: (A) a map of Vietnam indicating the location of Hanoi, and (B) the locations of Van Hoa and Yen Bai communes in Hanoi

in each commune reported to the commune offices. These numbers were accessed based on availability from the field.

Milk samples were collected from lactating dairy cows exhibiting no obvious clinical symptoms of mastitis and no visible changes in milk composition. Samples were taken from $60 \%$ of the delivered cows at all of the studied farms. The first few streams were discarded. Samples from each of the four quarters were collected into the same bottle and defined as a single milk sample for this study.

At each visit, an interview with the questionnaire was administered to obtain information regarding dairy management and hygiene awareness as well as cattle health outcomes. Occurrences of mastitis and repeat breeding within the previous three months were queried for each individual cow. The degree of each farm's sanitation was graded on a scale of 1-4 (1: cleanest to 4: dirtiest) for three parts of each cow: legs, udder, and flank and upper leg
(Reinemann, 2007). The average score of the three parts for all cattle was recorded as the farm's hygiene score. Farmers were also asked to rate their level of awareness regarding diseases common in cows, including mastitis and repeat breeding, using a Likert scale, with 0 indicating no problem and 5 indicating a significant problem.

Informed consent was obtained from all the farmers participating in this study at the time the interview was performed. This study did not involve invasive treatments in animals or collection of personal information through interviews. All interviewing and sampling procedures adopted in this study were approved by the ethics committee of Rakuno Gakuen University, Japan (approval number: 16-8).

\section{Laboratory examinations}

Causal organisms of sub-clinical mastitis were isolated from the collected milk samples. Microbial species isolated included coagulase- 
positive staphylococci (CPS) other than S. aureus, S. agalactiae, Escherichia coli, and Klebsiella spp. Cows were categorized as having sub-clinical mastitis if they presented no apparent symptoms but their milk samples were positive for bacterial growth. Milk samples were streaked on sheep blood agar plates, which support the growth of various types of bacteria. The plates were incubated at $37^{\circ} \mathrm{C}$ overnight. Three major bacterial colonies on each agar plate were selected and sub-cultured on blood agar plates. Biochemical tests were used for species identification, including the hemolysis, CAMP, catalase, and tube coagulase tests. Bacterial isolates that produced no hemolysis were subjected to biochemical testing for confirmation as E. coli or Klebsiella spp. Staphylococcus hyicus produces no hemolysis on blood agar, but $S$. aureus, S. intermedius, and S. agalactiae exhibit $\beta$-hemolysis. Therefore, these bacteria were classified based on the catalase test, which is primarily used to distinguish gram-positive bacteria. The results obtained with the catalase and coagulase tests were confirmed by PCR for $S$. aureus species-specific genes using the forward primer 5'-GCG ATT GAT GGT GAT ACG GTT-3' and the reverse primer 5'-AGC CAA GCC TTG ACG AAC TAA AGC-3' (Sasaki et al., 2010).

\section{Statistical analysis}

Data were digitized in a Microsoft Access ${ }^{\circledR}$ Database and analyzed using $\mathrm{R}$ statistical software $(\mathrm{R} \times 64$, version 3.0 .3$)$ for Windows ${ }^{\circledR}$. The association between insurance subscription and the aforementioned disease occurrence/ management practices was analyzed at the farm level. A farm was denoted as positive for mastitis/repeat breeding if at least one animal with the problem was reported or diagnosed by microbiological testing. These occurrences $(0=$ negative and $1=$ positive $)$ were used as response variables, and the other data collected in the questionnaire were used as explanatory variables. Each variable was analyzed using the Wilcoxon rank-sum, chisquare, or Fisher's exact test, depending on the data structure.

\section{Results}

\section{Farm characteristics}

All of the farms in the study were small scale ( 1 to 22 cows per farm), and $88.7 \%$ (86/97) of the farms had less than 10 cows. Table 1 shows the number of farms, milk samples, and mean herd sizes of the insured and non-insured farms. All cattle were housed on concrete surfaces. Cattle were fed raw grass and purchased concentrate at both insured and non-insured farms. There were no significant differences between insured and non-insured farms in any of the characteristics examined (Figure 2).

\section{Cumulative incidence of clinical mastitis and prevalence of sub-clinical mastitis}

In the questionnaire survey, factors related to mastitis were investigated using data from 95 farms, as two farms did not provide information regarding the incidence of mastitis in the interviews. The three-month cumulative incidence of clinical mastitis, calculated from the questionnaire survey results, was $22.1 \%$ (21/95) at the farm level. In addition, at the individual level, the incidence was $8.4 \%$ (29/344, health information regarding clinical mastitis was not obtained for 85 cows).

In the laboratory analyses, the prevalence of sub-clinical mastitis was $11.1 \%(17 / 153)$ at the individual level, and the prevalence at the farm level was $12.4 \%$ (12/97). Two samples had mixed infections involving S. agalactiae and S. aureus, and of the 19 isolates, these bacteria accounted for $57.9 \%$ (11 isolates) and $21.1 \%$ (4 isolates), respectively. There were three CPS isolates other than S.aureus, and these contagious mastitis-causing bacteria (S. agalactiae and CPS) accounted for $94.7 \%$ (18/19). One Klebsiella isolate causing environmental mastitis was detected; however, $E$. coli was not detected. Sub-clinical mastitis was found at 12 farms; thereby, both clinical mastitis and sub-clinical mastitis were identified in cattle at one farm.

Clinical and/or sub-clinical mastitic cattle were present on $33.0 \%$ (95\% CI: 24.0-43.4, 32/97) of the farms, based on the questionnaire survey and 
Influence of Mastitis and Repeat Breeding Incidence on Participation in the Animal Insurance Program

laboratory examination results. The proportion of farms with mastitic cattle was not significantly different between insured $(34.2 \%, 13 / 38)$ and noninsured farms $(32.2 \%, 19 / 59, \mathrm{p}=1)$. There was also no significant difference in terms of the incidence rates of clinical and/or sub-clinical mastitis between insured $(29.3 \%, 17 / 58)$ and noninsured farms $(22.1 \%, 21 / 95, \mathrm{x} 2=0.6527, \mathrm{p}=0.42)$.

Table 1. Farm size and number of milk samples

\begin{tabular}{lccccc}
\hline Insurance & $\begin{array}{c}\text { Farms in } \\
\text { Yen Bai }\end{array}$ & $\begin{array}{c}\text { Farms in } \\
\text { Van Hoa }\end{array}$ & $\begin{array}{c}\text { Mean herd size } \\
\text { (range) }\end{array}$ & Adult cows & $\begin{array}{c}\text { Milk samples } \\
\text { (sampling fraction) }\end{array}$ \\
\hline I & 16 & 22 & $5.4(2-22)$ & 153 & $58(37.9 \%)$ \\
Non-insured & 33 & 26 & $5.9(1-13)$ & 239 & $95(39.7 \%)$ \\
Total & 49 & 48 & $5.7(1-22)$ & 392 & $153(39.0 \%)$ \\
\hline
\end{tabular}
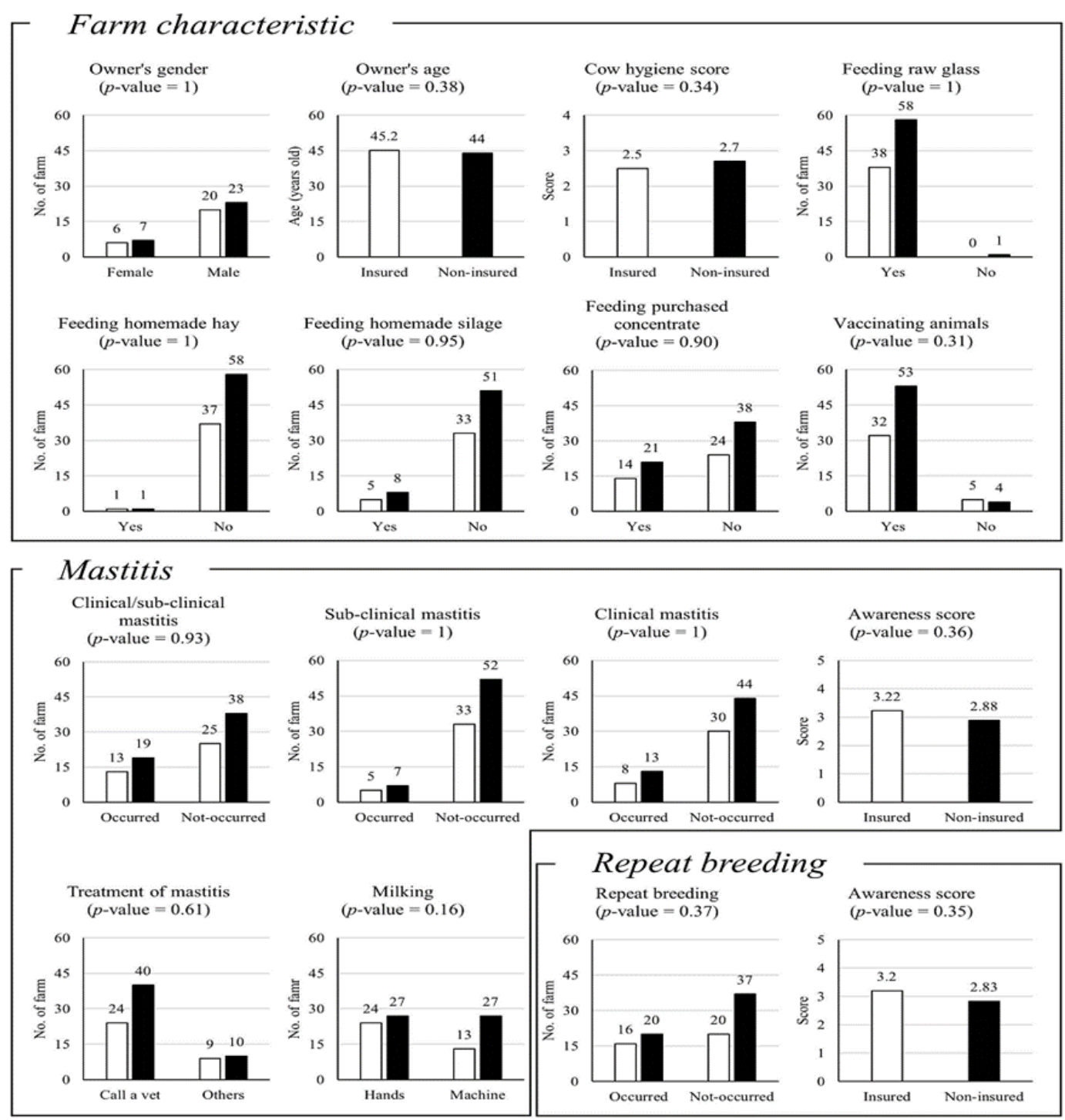

Insured farm Non-insured farm

Figure 2. Statistical analysis results 
The average scores regarding farmers' awareness of mastitis were not significantly different between insured (3.22, 95\% CI: 3.0-4.5) and non-insured farms $(2.88,95 \%$ CI: $2.75-4.00, \mathrm{p}$ $=0.36)$. For the treatment of mastitis, $77 \%(64 / 83)$ of farmers called a veterinarian, whereas $23 \%$ (19/83) of farmers called other staff or treated the disease themselves. There was no significant difference between insured and non-insured farms in terms of factors relating to mastitis.

\section{Incidence and farmers' awareness of repeat breeding}

Ninety-three farmers answered the questions relating to reproductive difficulties, and the results indicated that repeat breeding occurred at $38.7 \%(36 / 93)$ of the studied farms. The proportion of farms with these reproductionrelated problems did not differ between insured $(44.4 \%, 16 / 36)$ and non-insured farms $(35.1 \%$, 20/57, $\mathrm{x} 2=0.47, \mathrm{df}=1, \mathrm{p}=0.49$ ). In addition, the average score of awareness of repeat breeding was not significantly different between insured (3.2, 95\% CI: 2.5-4.5) and non-insured farms (2.8, 95\% CI: $2.50-3.75, \mathrm{p}=0.35)$. Artificial insemination was used at all the farms.

\section{Discussion}

The main purpose of this study was to test the hypothesis that Vietnamese dairy farmers were motivated to participate in an animal insurance program due to experience with chronic production diseases, even though the program targeted acute infectious diseases. For that reason, two economically important diseases, mastitis and repeat breeding, were examined.

There were no significant differences in terms of farm size, feed management, vaccination, or disease occurrence between insured and non-insured farms in this study. In laboratory analyses, contagious pathogens associated with mastitis were identified much more frequently than environmental pathogens, indicating that the milking method is more important than environmental factors in efforts to control the spread of mastitis in this region. In this study, the p-values of most factors were greater than 0.30 , but the p-value associated with the milking method was relatively lower $(\mathrm{p}=$ 0.16 ), and farms with milking machines tended not to participate in the insurance program. Milking by machine is a sign of intensive dairy farming, and economically developed farmers may evaluate the significance of the program carefully. Another study carried out during the same period but with different farmers (Kono et al., 2017) showed that a farmer's decision to purchase animal insurance was affected by the absence of a trustworthy person nearby and a low rate of time discounting. Such sociological factors seemed to be more significant determinants of insurance participation by dairy farmers than disease burden.

\section{Conclusions}

This study found that the incidence of two important production diseases, mastitis and repeat breeding, was not the primary determining factor for the decision to participate in the animal insurance program in $\mathrm{Ba} \mathrm{Vi}$, Vietnam. The risk factors for mastitis and repeat breeding in Vietnam will be published elsewhere.

\section{Acknowledgements}

This study was supported by KAKENHI Grant Number 25304035, from the Ministry of Education, Culture, Sports, Science, and Technology of Japan.

\section{Conflicts of Interest}

The authors declare no conflicts of interest.

\section{References}

Bartlett P. C., Kirk J. H. \& Mather E. C. (1986). Repeated insemination in Michigan Holstein-Friesian cattle: Incidence, descriptive epidemiology and estimated economic impact. Theriogenology. 26: 309-322.

Duc D. M. (2017). Agricultural insurance in Vietnam: pilot programme and pre-conditions for a public-private partnership approach. Asia Pacific Journal of Public Administration, 39(1): 63-71.

Dohoo I., Wayne M. \& Stryhn H. (2014). Veterinary Epidemiologic Research ( $2^{\text {nd }}$ ed.). VER Inc. Charlottetown. 
Food and Agricultural Organization (2013). Food Outlook, November 2013: Global Market Analysis. Retrieved from http://www.fao.org/docrep/019/i3473e/i3473e. pdf on July 23, 2017.

Garcia O., Hemme T., Nho L. T., \& Huong Tra H. T. (2006). The economics of milk production in Hanoi, Vietnam, with particular emphasis on small-scale producers. A Living from Livestock. 54.

Grant R. J. \& Albright J. L. (1995). Feeding behavior and management factors during the transition period in dairy cattle. Journal of Animal Science. 73: 2791-803.

Gustafsson H. \& Emanuelson U. (2002). Characterisation of the repeat breeding syndrome in Swedish dairy cattle. Acta Veterinaria Scandinavica. 43: 115-125.

Hogeveen H., Huijps K., \& Lam T. J. G. M. (2011). Economic aspects of mastitis: new developments. New Zealand Veterinary Journal. 59: 16-23.

Lam V. (2011). Milk production on smallholder dairy cattle farms in southern Vietnam. Doctoral thesis, Swedish University of Agricultural Sciences, Uppsala.

Nguyen-Kien C., Khanh N. V. \& Hanzen C. (2017). Study on reproductive performance of Holstein $\mathrm{x}$ Lai Sind crossbred dairy heifers and cows at smallholdings in Ho Chi Minh City, Vietnam. Tropical Animal Health and Production. 49: 483-489.

Japanese Ministry of Agriculture Forestry \& Fisheries (2010). Overview and structure of livestock insurance scheme. Retrieved from http://www.maff.go.jp/j/ council/seisaku/kyosai/bukai/05/pdf/data4.pdf on June 21, 2019 (in Japanese).

Kadowaki H., Kayano T., Tobinaga T., Tsutsumi A., Watari M. \& Makita K. (2016). Analysis of factors associated with hesitation to restart farming after depopulation of animals due to 2010 foot-and-mouth disease epidemic in Japan. Journal of Veterinary Medical Science. 78: 1251-1259.

Keefe G. P. (1997). Streptococcus agalactiae mastitis: A review. Canadian Veterinary Journal. 38: 429-437.

Kono H., Kubota S., Sembokuya Y., Makita K., Nishida T., Hien N. \& Hai T. (2017). Animal Insurance and Farmer's Behavior in Vietnam. Asian Journal of Agricultural Extension, Economics and Sociology. 16: $1-12$.

LeBlanc S. J., Lissemore K. D., Kelton D. F., Duffield T.
F. \& Leslie K. E. (2006). Major advances in disease prevention in dairy cattle. Journal of Dairy Science. 89: 1267-1279.

Östensson K., Lam V., Sjögren N. \& Wredle E. (2013). Prevalence of subclinical mastitis and isolated udder pathogens in dairy cows in Southern Vietnam. Tropical Animal Health and Production. 45: 979-986.

Poolkhet C., Makita K., Thongratsakul S. \& Leelehapongsathon K. (2018). Exponential random graph models to evaluate the movement of backyard chickens after the avian influenza crisis in 2004-2005, Thailand. Preventive Veterinary Medicine. 158: 71-77.

Reinemann D. J. (2007). Clean Cows Mean Less Mastitis. Retrieved from http://www.medvet.umontreal.ca /rcrmb/dynamiques/PDF_AN/Management/CleanCo wsLessMastitis.pdf on June 21, 2019.

Roberson J. R., Fox L. K., Hancock D. D., Gay C. C. \& Besser T. E. (1994). Coagulase-Positive Staphylococcus Intramammary Infections in Primiparous Dairy Cows. Journal of Dairy Science. 77: 958-969.

Sasaki T., Tsubakishita S., Tanaka Y., Sakusabe A., Ohtsuka M., Hirotaki S., Kawakami T., Fukata T. \& Hiramatsu K. (2010). Multiplex-PCR method for species identification of coagulase-positive staphylococci. Journal of Clinical Microbiology. 48: 765-769.

Suzuki K., Kanameda M., Inui K., Ogawa T., Nguyen V. K., Dang T. T. S., \& Pfeiffer D. U. (2006). A longitudinal study to identify constraints to dairy cattle health and production in rural smallholder communities in Northern Vietnam. Research in Veterinary Science. 81: 177-184.

Vietnam News (2018). Poor farmers to be supported with up to 90 per cent of agricultural insurance. Retrieved from https://vietnamnews.vn/society/427097/poorfarmers-to-be-supported-with-up-to-90-per-cent-ofagricultural-insurance.html\#DdT7HFzjAM8ahwWt. 97 on October 24, 2019.

Vu L. T., Long N. T., Brito B., Stenfeldt C., Phuong N. T., Hoang B. H., Pauszek S. J., Hartwig E. J., Smoliga G. R., Vu P. P., Quang L. T. V, Hung V. V., Tho N. D., Dong P. V, Minh P. Q., Bertram M., Fish I. H., Rodriguez L. L., Dung D. H. \& Arzt J. (2017). First detection of foot-and-mouth disease virus O/Ind2001d in Vietnam. PloS one. 12: e0177361. 